\title{
Measurements in Concentrated Sun using a Remote Controlled Robot
}

\author{
http://dx.doi.org/10.3991/ijoe.v9iS3.2544
}

\author{
Dan Floroian ${ }^{1}$, Laura Floroian ${ }^{1}$, Rachamim Rubin ${ }^{2}$, Doron Lieberman ${ }^{2}$, \\ Petru Cotfas ${ }^{1}$, Daniel Cotfas ${ }^{1}$, Doru Ursutiu ${ }^{1}$, Cornel Samoila ${ }^{1}$ \\ ${ }^{1}$ Transilvania University of Brasov, Romania \\ ${ }^{2}$ Weizmann Institute of Science, Rehovot, Israel
}

\begin{abstract}
Nowdays, using the concentrated sunlight is a big issue because the amount of energy is very high and the light is concentrated in a very small area. The main problem in this situation is the heating, and in order to make safe measurements a remote controlled robot is needed. After that, a remote controlled robot will assume the duty of protect the measured sample and to expose it for a precise time to the concentrated sun in order to reduce heating of the sample. For easy operating, and for automatize the process, all the duties, starting with initial conditions, continuing with triggering the measurements, and conditioning the signals and finalizing with data saving must be assured by the robot.
\end{abstract}

Index Terms-automatic measurements, concentrated sun, remote control, robotic arm.

\section{INTRODUCTION}

A very promising renewable source of energy is concentrated solar power (CSP) obtained from solar concentrators. The solar North Africa continent and also Mediterranean countries of the European Union have a big resource of sun. The best known its application is electricity generation through thermodynamic cycles, but other applications have also been demonstrated, such as solar fuels and production of hydrogen, research in advanced materials and water treatment. Unfortunately the less studied process is the subject of solar cells in solar concentrator systems. Because of the boom of photovoltaic cells recorded in last years, we have in present many types of photovoltaic cells with different efficiencies and we don't have enough studies focusing of concentrated sun effects on these cells. The areas of applications is quite large starting with special equipment used on satellites and continuing with powerful power stations placed in suitable places on earth.

The concentrator makes it possible to focus sunlight falling on a large area onto a very small area. This increases the intensity of the solar radiation, leading to greater power falling on the area of focus. The solar intensity (or concentration ratio) is determined by the ratio of the area of incident sunlight to the area that it is focused on. Concentration ratios of the thousands have been achieved [1].

The use of solar concentrators creates the potential for the production of less expensive solar panels using high efficiency multi-junction cells or mono-crystalline cells. In general the materials used to build concentrators are less expensive than photovoltaic cells. The concentrator takes up most of the area of a concentrator system, and only a small amount of photovoltaic material is needed. Concentrator panels could reduce the cost per watt ratio in the point where solar power is an economical alternative.

Photovoltaic technology was developing since 1954 [1], but it is not a widely used source for the production of electricity yet. This is because the amount of produced power compared to the cost of solar panels makes their purchase uneconomical for most buyers. Significant gains have been made in the cost per watt ratio since the 1970's reducing the price from $\$ 70$ per Watt to under $\$ 4$ per Watt today [2]. However, this ratio needs to be reduced further before photovoltaic technology becomes a viable resource. Now the market is dominated by two types of photovoltaic cells. The silicon cells are the most widely used; they constitute $86 \%$ of the current market [2]. Mono crystalline cell technology is well developed in our days because it uses the same manufacturing techniques that are used in the electronics semiconductor industry. In this process, a mono silicon crystal is grown and then sliced into thin wafers. This produces identical and efficiently cells those are up to 15-17 percent efficient. However, the process is quite time consuming and pretty expensive. A less expensive but not so efficiently technique is the production of polycrystalline silicon cells. In this process molten silicon is poured into molds and allowed to cool, then sliced into wafers similar to those in the single crystal method. This method is very popular in these days. Many small silicon crystals are formed in the mold instead of one large one as in the single crystalline cell. This results in the efficiency of the polycrystalline cell being lower, between 11 and 14 percent [2].

The remaining $14 \%$ of the PV market is based on thin film semiconductors make up [2]. For this process, a thin film of semiconductor material (most often amorphous silicon) is deposited on an ordinary substrate such as glass or composite. This process is fast and uses much less material because the silicon layer is about one micron thick. Because thin film has no crystalline structure, it results in efficiencies of only six to eight percent. The low cost of production faces the poor efficiency, and thin films could be relevant in the future of the photovoltaic market.

Now days, efficient cells have been developed, but due to their high price, they are used mainly in research or in space technology. The most promising high-efficiency cell is the multi-junction cell. Multi-junction cells are several layers of photovoltaic cells stacked on top of one another. Each successive layer has lower band gap energy, allowing for the absorption of a wider range of the spectrum. 


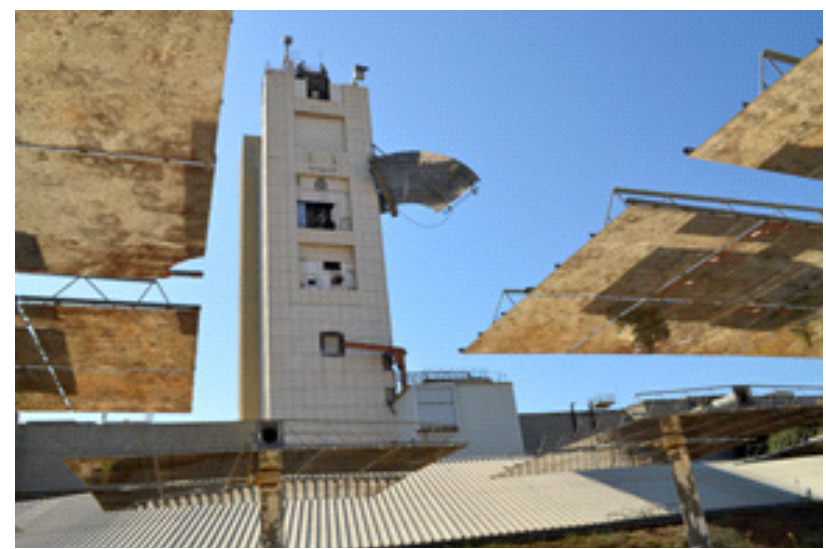

Figure 1. Solar tower

Boeing holds the current record for the efficiency of a multi-junction cell at 34.2 percent [1]. This is more than twice the efficiency of cells currently on the market, and efforts are being made to increase the efficiency up to 40 percent.

Is not practical to build a solar panel out of multijunction cells because they are so expensive in the present time. However, a solar concentrating system utilizing multi-junction cells could be cost effective. At present there are many different types of concentrators that could be used in a photovoltaic system. These concentrators can be divided into two different groups: reflecting concentrators and lenses. In the former group the most common concentrators are flat plate mirrors, spherical and parabolic mirrors, and cylindrical trough collectors. The most prominent lens is the Fresnel lens. It was developed in 1822 for use in lighthouses and can achieve high concentration ratios [1]. Newer lenses such as Aspheric lenses and TIR (Transmission, total Internal reflection, Refraction) lenses can be used together to achieve concentration ratios of over 300 while being only $2 \mathrm{~cm}$ thick.

The issue of using concentrators is not a simple problem because most concentrators rely on being directly focused on the sun; any deviation causes a severe drop in the concentration ratio. Some of the collected power must be used to run a tracking system. Also, though it seems that the power converted by a solar cell would increase indefinitely with increasing illumination, is not the case. As the intensity of illumination increases, the solar cell heats up. It is a well-documented fact that the efficiency of solar cells decreases as the temperature of the cell increases. The loss in efficiency is about $10 \%$ for every 25 $\mathrm{K}$ increase in temperature [3], although the exact loss in efficiency depends on the specific cell [4].

Also a too high temperature that can be achieved in such cases can damage the cell's integrity. This is particularly important in dish and tower systems where a maximum uniform flux may be difficult to achieve. A reliable cooling system is a very crucial issue, since any failure in the cooling system can lead to catastrophic results from cell damage to fire hazards in extreme cases. Coolants should be selected to minimize the health and environmental hazards. A simple design can help to reduce the maintenance costs.

Many cooling techniques have been proposed for cooling of solar PV cells under high concentration. These techniques aim to attain low and uniform cell temperature using simple designs. Royne et al. [5] have studied the

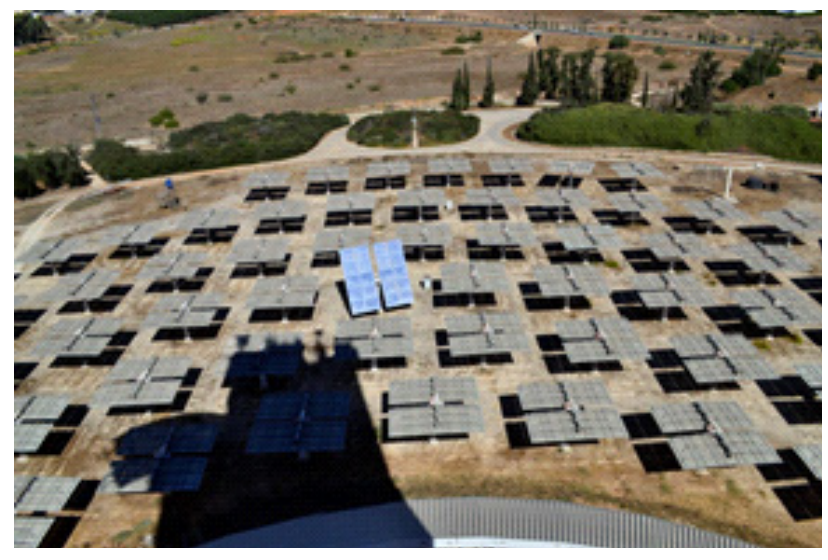

Figure 2. Heliostats field

cooling of densely packed photovoltaic cells under high concentration using a jet impingement technique. An optimization is performed for selecting the jet diameter to accommodate various cell illumination conditions. The jet cooling technique results in non-uniform heat transfer from the cells surface, although this non-uniformity didn't affect the electrical output strongly. Passive cooling techniques are employed with linear concentrators like the trough system where the concentration ratio is low, around 20 suns. Akbarzadeh et al. [6] used a heat pipe type passive cooling system with R22 refrigerant as a cooling medium to cool down a solar trough concentrated PV system. Using channels attached to the back of the PV cell, Lasich [7] managed to keep the cells at temperatures around $40^{\circ} \mathrm{C}$. Recently was checked the validity and applicability for a new cooling technique, the immersion cooling [8].

This paper presents a method for experimental solar cells characterization into high concentrated solar light without using another cooling system than simple classical remove of heat from the back (opposite surface of the incident flux exposed surface) of the cell.

Our method consists of rapid measurement of cell current and voltage in about one second and immediately covering the cell for its protection against powerful solar radiation using a remote controlled automatic device.

The concentrated sun light was supplied by Solar Research Facility Unit from Weizmann Institute of Science, Rehovot, Israel.

The Solar Research Facilities Unit has a very sophisticated solar tower with a north field of 64 heliostats, $56 \mathrm{~m}^{2}$ each, which on a bright day can collect $3 \mathrm{MW}$ of solar radiation in total. The tower has 4 vertical experimental levels, 3 indoor and one outdoor on the roof for which special safety precautions are required for the tests. In addition, the facility has a unique $0.5 \mathrm{MW}$ beam-down facility, which has no equal in any other solar research facility in the world (Fig. 1).

For our work we used one heliostat (Fig. 2) to obtain concentrated sunlight from 700 to 1000 suns and the whole experiment was made in order to obtain the parameters of different types of solar cells (commercial and manufactured). The determination of solar cells and panels' parameters is important for researchers, manufacturers and users as well as for stand-alone or on grid solar panels designers. 


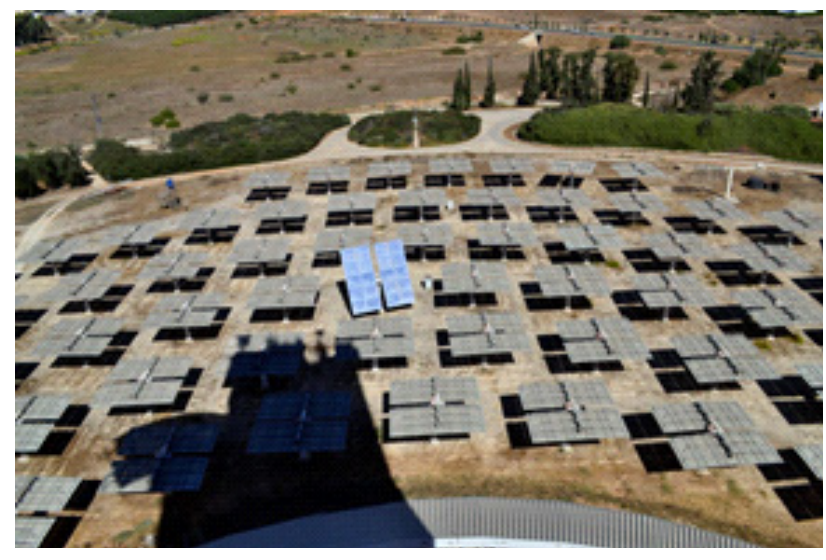

Figure 3. Heliostats field

There are several methods to characterize and determine the parameters of solar cells and panels. Most of these are based on using the current-voltage characteristic $\mathrm{I}-\mathrm{V}$. The parameters that can be determined using one or more I-V characteristics are: $V_{\mathrm{oc}}$ - open circuit voltage, $\mathrm{I}_{\mathrm{sc}}$ - short circuit current, $P_{\max }$ - maximum power, $R_{\text {sh }}$ - shunt resistance, $\mathrm{R}_{\mathrm{s}}$ - series resistance, $\mathrm{m}$ - ideality factor of diode, $\mathrm{FF}$ - fill factor, $\mathrm{I}_{\mathrm{o}}$ - reverse saturation current and $\eta$ efficiency.

A simple and quick method to characterize the solar cells and panels is the plotting of the I-V characteristic and its non-linear fitting using the one diode model, which mathematically describes the behavior of photovoltaic cells [9]. The approximation used for the fitting is

$$
V_{\max } \gg I_{\max } R_{s}
$$

and thus the equation describing the one diode model becoming explicit (2).

$$
l=I_{s C}-I_{9}\left(\frac{a r}{m k T}-1\right)-\frac{y}{R_{a h}}
$$

The fitting ensures the determination of the following parameters: $I_{o} I_{s c}, R_{s h}, m, P_{\max }$ and implicitly $\eta$ and FF can be determined using the P-V (power- voltage) characteristic [10].

\section{ROBOTIC ARM}

In order to achieve information at high concentration of solar radiation, is necessary to build a special remote controlled robotic device because the temperature on cell is around $1000{ }^{\circ} \mathrm{C}$ and ordinary solar (not specially very high temperature designed) cells or electrical contacts couldn't resist. This remote controlled robotic device is constructed with two parts that work together: an I-V module and a robotic arm.

$\mathrm{I}-\mathrm{V}$ module is based on high capacitors (minimum 0.4 Farads) which also has very low internal resistance. In this situation, we are able to obtain characteristics in less than 1 second and to expose the cell very shortly in order to reduce cell heating. First, the robot shortcuts the capacitor in order to obtain a fresh measurement; second, it moves the robotic arm to uncover the solar cell and triggers the measurements when the robotic arm is in position; in the third step it covers the solar cell and in the last operation saves the data into a file. The robot works under control of 12 bits National Instruments acquisition board which is also controlled by a program in the graphical program- ming language Lab VIEW. The Lab View program was created to interface both with the user and the computer.

At beginning the user start program computer and this program send a message to the robot using NI board. At the moment of receiving this message, the robot will close a relay in order to produce a discharge of high capacity capacitor with the purpose of recording only the voltage acquired in measurement period of time. After this process is completed, the computer program will be noticed (again using NI boards) and it will pass to the next step, respectively sending another message to the robot. This second message will instruct robot to move the arm in order to uncover the sample. When the movement is completed, a sensor is activated and the computer program receives a message from controller program thru NI board. At this time, the NI board is activated in acquisition mode and the measurement starts. This measurement is maintained for $1 \mathrm{~s}$ and after that is stopped. During this period of time 1000 samples are collected. The length of acquisition time (and of course the number of samples) is determined in computer program also.

After measurement, the computer program flow to the next step and sends a stop message to acquisition board and to the robot also. At receiving the message, the robot will move back its arm and will cover back the sample. After this process is completed, the computer program will receive a message for "job done" confirmation. In every step of the described process an error tag is positioned in case of malfunction and the computer program will show an error message in order to clarify the occurred problem.

The controller is based on ATMEGA microcontroller and all parallel ports are used in order to assure all necessary functions. Furthermore it could be said that in the same time communications with computer is provided and also communication with acquisition board, with sensors and I-V module are supplied.

\section{I-V DRIVER AND LABVIEW PROGRAM}

There are various devices and techniques used by research for raising the solar cells I-V characteristics.

Among the most widely spread is the use of the electronic load.

There are three methods to operate an electronic load: the constant current, the constant voltage, and the constant resistance; from these it is advisable to use the constant voltage, when the voltage is varied with the desired step and the current on the cell is measured.

But the use of the electronic load presents two disadvantages. One of them is the duration of the I-V characteristic raising and the other one is the high cost. The duration is in general of tens of seconds, enough to modify the cells temperature. This fact leads to variations of solar cells parameters during the measurements.

To avoid this temperature variation a thermostat can be used to maintain the temperature constant during measurements.

However an impediment appears because the solar panels as well as the solar cells are encapsulated, this making the use of the thermostat more difficult.

To remove all these drawbacks our I-V characteristic module (Fig.4) is designed using the charging schematics of a large capacitor. The functioning principle that under- 
lies this technique is measuring the current and the voltage during the charging of the capacitor. The time duration of raising the I-V characteristic of the solar cell is in hundredths of a second range, fact that ensures that the cell temperature variation during measurement is practically zero. Another advantage is the high number of $(\mathrm{V}, \mathrm{I})$ points which can be acquisitioned. The large number of points allows a very good fitting of the I-V characteristic.

In aim of achieve a short charging time and because we need a large capacity, it is mandatory to have a very small internal resistance $(\approx 40 \mathrm{~m} \Omega)$. Also in the same direction it is necessary that the resistance for current measurement to have a very small resistance $(20 \mathrm{~m} \Omega)$.

Furthermore the voltage on the resistance is very small and needs to be amplified before sending the signals to the measurement equipment.

The conditional amplifiers are conducted by microcontroller on robotic arm and could be programed in order to amplify with 10 or 100 . After that, the signal could be passed on shielded wires to the acquisition board. For a good insulation digital signals are separated from analogical using different cables, also shielded.

For highly accurate measurements a National Instruments NI 6009 acquisition board was chosen, with 8/4 analog input (AI) channels in the configuration SingleEnded Channels/Differential Channels with a sampling rate of $48 \mathrm{kS} / \mathrm{s}$ and 14 bits resolution, 12 Digital Input / Output (DIO), two analog input channels AO of a 12 bits resolution and a counter. The connection of the board to a laptop is realized by a USB connection.

The program for data acquisition is made using the visual VIs provided by default and configuring it. The only special issue is that signals are forced in 1 and lowered in 0 only in time of the action in order to prevent the perturbations, especially because of electromagnetics perturbations in this special experiment. The user interface is presented in figure 5 .

Software applications were created in the graphical programming language LabVIEW to control the circuit, to automatically raise the I-V characteristic and to determine the parameters. The applications were then compiled into one complex application. The application is created on tabs, see Figure 5. The first tab "Raising of I-V characteristic" permits the control of the acquisition board, the control of the capacitor, the plotting of the I-V characteristic and the saving of the data. The tab "Determination of parameters" permits the import of the processing file and the determination of the interest cells parameters using the method of the nonlinear fitting. The other methods to determine the parameters of the solar cells and panels described in the theoretical considerations were implemented as sub-tabs in the tab "Applications".

\section{RESULTS}

Using the method just we studied different types of solar cell behavior in concentrated light:

- at different solar illumination level (700, 900 and 1000 suns)

- at different temperatures (24 and 30 degrees Celsius)

- at different wavelengths in UV-VIS-IR range: 461 $\mathrm{nm}, 508 \mathrm{~nm}, 591 \mathrm{~nm}, 623 \mathrm{~nm}, 672 \mathrm{~nm}, 766 \mathrm{~nm}, 773$ $\mathrm{nm}, 793 \mathrm{~nm}, 795 \mathrm{~nm}$.

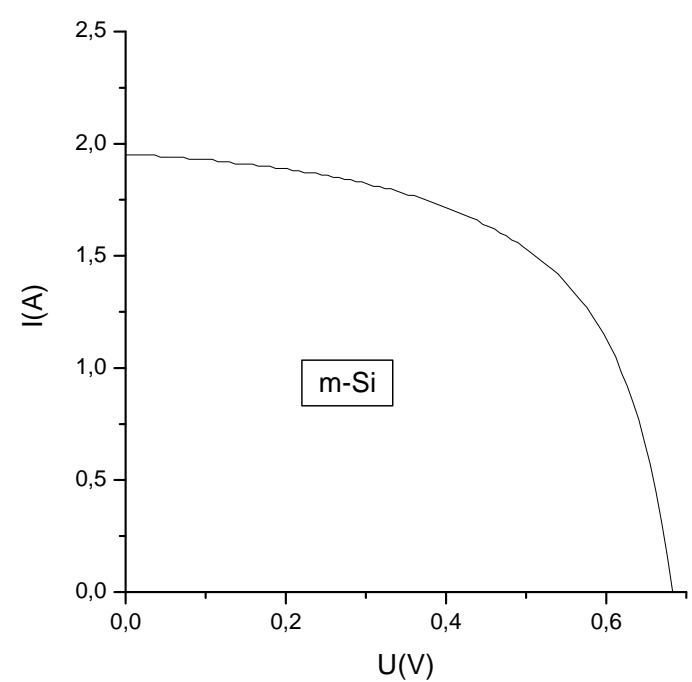

Figure 4. I-V Characteristic

We founded correlation between the laser deposition parameters and the optical and electrical properties of the layers and also we determined properties and parameters of the solar cells under concentrated solar power: shortcircuit current, cell efficiency, open circuit potential, fill factor, maximum power, series and shunt resistance. We recorded simultaneously current intensity, voltage, flux and intensity of radiation using 4 channels of data acquisition plaque, for each solar cell.

An example of I-V characteristic of Si monocrystalline cell at 700 suns is presented in figure 6. It shows a different shape from one sun irradiation level, it has a high short circuit current and open circuit potential is $\mathrm{V}_{\mathrm{oc}}=0.659 \mathrm{~V}$. Table I shows the values of the cells parameters obtained at 700 suns using the method before described.

\section{CONCLUSIONS}

This paper presents a method for experimental solar cells characterization into high concentrated solar light without using another cooling system than simple classical remove of heat from the back (opposite surface of the incident flux exposed surface) of the cell.

Our method consists of rapid measurement of cell current and voltage in about one second and immediately covering the cell for its protection against powerful solar radiation using a remote controlled automatic device.

The remote controlled robot was able to complete its mission in protecting the solar cells and in raising the I-V characteristics of 4 different solar cells and in more than 400 different experiments.

The whole system (which brings together digital electronics, analog electronics, data acquisition board, a laptop and LabView program) worked fine even in strong electromagnetically perturbation.

\section{ACKNOWLEDGMENT}

Financial support by the Access to Research Infrastructures activity in the 7th Framework Programme of the EU (SFERA Grant Agreement n. 228296) is gratefully acknowledged.

Also the Romanian authors acknowledge with thanks the support of Solar Research Facility Unit from Weizmann Institute of Science, Rehovot, Israel, where all the measurements were performed. 


\section{MEASUREMENTS IN CONCENTRATED SUN USING A REMOTE CONTROLLED RoBOT}

\section{REFERENCES}

[1] B. Parida, S. Iniyan, R. Goic, "A review of solar photovoltaic technologies", Renewable and Sustainable Energy Reviews, vol. 15, Issue 3, pp. 1625-1636, 2011. http://dx.doi.org/10.1016/ j.rser.2010.11.032

[2] F. Dinçer, "The analysis on photovoltaic electricity generation status, potential and policies of the leading countries in solar energy", Renewable and Sustainable Energy Reviews, vol. 15, Issue 1, pp. 713-720, 2011. http://dx.doi.org/10.1016/j.rser.2010.09.026

[3] M.A. Mosalam Shaltout, M.M. El-Nicklawy, A.F. Hassan, U.A. Rahoma, M. Sabry, "The temperature dependence of the spectral and efficiency behavior of Si solar cell under low concentrated solar radiation" Renewable Energy, vol.21, pp. 445-458, 2000. http://dx.doi.org/10.1016/S0960-1481(00)00075-6

[4] E. Radziemska, E. Kleugmann, "Thermally Affected Parameters of the Current-Voltage Characteristics of Silicon Photocell" Energy Conversion and Management vol.43, pp. 1889-1900, 2002. http://dx.doi.org/10.1016/S0196-8904(01)00132-7

[5] A. Royne, C.J. Dey, "Design of a jet impingement cooling device for densely packed PV cells under high concentration", Solar energy, vol. 81, pp. 1014-1024, 2007. http://dx.doi.org/10.1016/ j.solener.2006.11.015

[6] A. Akbarzadeh, R. Singh, H. Fan, "Electric power generation by thermoelectric cells and parabolic solar concentrators", Proceedings of the Solar09, the 47th ANZSES Annual Conference, 29 Sept - 2 Oct, 2009, Townsville, Queensland, Australia.
[7] J.B. Lasich, "Cooling circuit for receiver of solar radiation", Patent WO02080286, Australia, 2002

[8] L. Zhu, R.F Boehm, Y. Wang, C. Halford, Y. Sun, "Water immersion cooling of PV cells in a high concentration system", Solar Energy Materials and Solar Cells, vol. 95, Issue 2, pp. 538545, 2011. http://dx.doi.org/10.1016/j.solmat.2010.08.037

[9] D.T. Cotfas, P.A. Cotfas, S. Kaplanis, D. Ursutiu, " Results on series and shunt resistances in a c-Si PV cell", J optoelectron adv m., vol. 10, pp. 3124-3130, 2008.

[10] D.T. Cotfas, P.A. Cotfas, D. Ursutiu, C. Samoila, "CurrentVoltage Characteristic Raising Techniques for Solar Cells. Comparisons and Applications", 12th International conference on optimization of electrical and electronic equipaments, Brasov, Romania, IEEE Xplore Digital Library, 2010.

\section{AUTHORS}

Dan Floroian, Laura Floroian, Petru Cotfas, Daniel Cotfas, Doru Ursutiu, and Cornel Samoila are with Transilvania University of Brasov, Romania.

Rachamim Rubin and Doron Lieberman are with the Weizmann Institute of Science, Rehovot, Israel.

This article is an extended and modified version of a paper presented at the International Conference on Remote Engineering \& Virtual Instrumentation (REV2012), held at University of Deusto, Bilbao, Spain, July 4-6, 2012. Received 01 March 2013. Published as resubmitted by the authors 20 March 2013. 